whereupon they switched to the intervention phase (add-on programme) sequentially and in randomised order. The add-on programme comprised structured individualized goal planning, motivational interviewing, a self-help booklet and four supportive follow-up phone calls the first five months after discharge. Data were collected on admission, discharge, 6 and 12 months after discharge. Primary outcome was health related quality of life (HR-QoL) measured by the individualized Patient Generated Index (PGI). The main statistical analysis was a linear repeated measures mixed model performed on the intention to treat population (all available data).

Results: 389 patients with various rheumatic diseases (SpA, RA, OA, and SLE) were included (table 1). A significant treatment effect of the add-on intervention on HR-QoL was found on discharge (mean difference $=3.32[95 \% \mathrm{Cl}: 0.27$, 6.37], $p=0.03$ ). There were no significant differences between the groups at 6 and 12 months. Treatment compliance was $94 \%$, and response rate $>80 \%$ at all time points. Both groups showed a positive effect of rehabilitation in terms of increased HR-QoL at discharge, which subsequently declined, although the values remained at higher levels after 6 and 12 months compared with baseline values (figure 1).

Table 1. Baseline characteristics of included patients $(n=389)$

\begin{tabular}{lcc}
\hline & Control group $(\mathrm{n}=195)$ & Intervention group $(\mathrm{n}=194)$ \\
\hline Age, yrs, mean (min, max) & $56.9(24,89)$ & $57.5(23,89)$ \\
Gender,, $\mathrm{n}(\%)$ & $127(65.1)$ & $147(75.8)$ \\
Disease duration, yrs, mean (SD) & $21.0(13.3)$ & $19.1(13.1)$ \\
Paid work, $\mathrm{n}(\%)$ & $69(35.8)$ & $76(39.4)$ \\
Using TNF-inhibitors, $\mathrm{n}(\%)$ & $44(22.6)$ & $39(20.1)$ \\
\hline
\end{tabular}

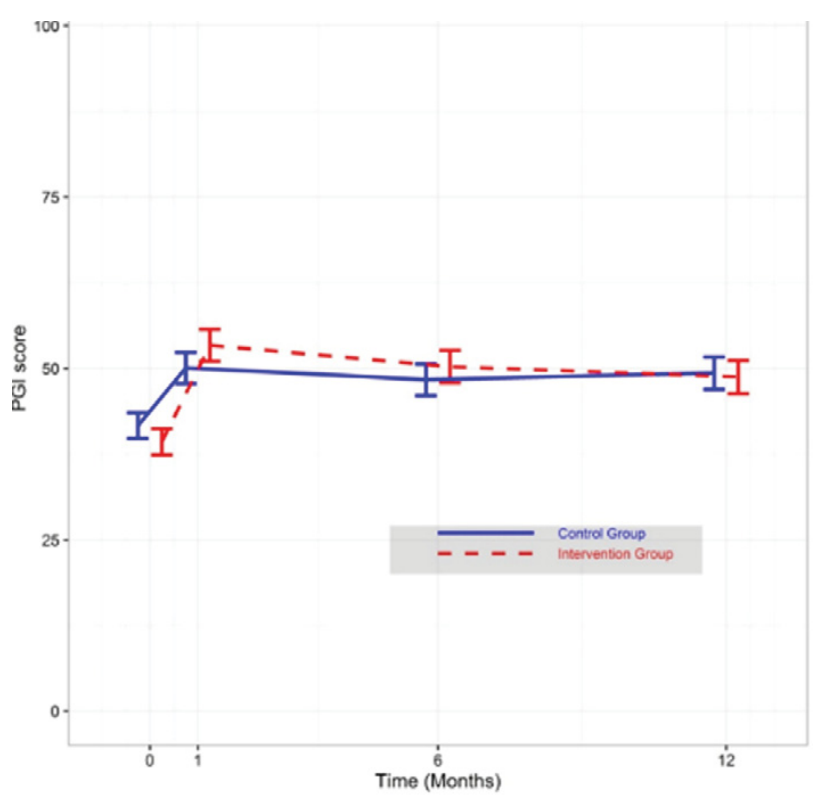

Figure 1. Health related quality of life in patients with rheumatic diseases measured by the Patient Generated Index (PGI, 0-100, 0 low HR-QoL). Vertical lines indicate the mean values (centre) with $95 \%$ confidence intervals at the four measurement time points: on admission to rehabilitation stay (baseline), at discharge, and six and twelve months after discharge. Horizontal lines show the fluctuating mean PGI values from baseline to 12 months after discharge.

Conclusions: The add-on intervention enhanced the short-term effect of rehabilitation with respect to HR-QoL, but did not prolong the effect as intended. The findings suggest that individualized structured goal planning should be considered important and relevant in rehabilitation of patients with rheumatic diseases. Although this study evaluated the effect of a complex intervention, and not the effect of single components, the results indicate that supportive telephone follow-up after discharge do not prolong the effect of rehabilitation. However, this study showed a longer lasting effect of rehabilitation in both the control- and intervention group, compared to previous reports.

Disclosure of Interest: None declared

DOI: 10.1136/annrheumdis-2017-eular.3383

\section{OP0256-HPR PHYSICAL ACTIVITY AND INACTIVITY IN PATIENTS WITH SYSTEMIC LUPUS ERYTHEMATOSUS}

C. Boström ${ }^{1,2}$, F. Russ ${ }^{1}$, F. Eriksson ${ }^{1}$, S. Pettersson ${ }^{2}$, I. Gunnarsson ${ }^{1,2}$, E. Svenungsson $1,2 .{ }^{1}$ Karolinska Institutet; ${ }^{2}$ Karolinska Universitetssjukhuset, Stockholm, Sweden

Background: Previous studies indicate that patients with systemic lupus erythematosus (SLE) have a reduced physical activity compared to controls and public health recommendations. However, most studies have used questionnaires without expressing the energy requirements for the activities.
Objectives: We investigated if self-reported physical activity, expressed in Metabolic Equivalent of Task (METs)-minutes/week, in patients with SLE is reduced. The aim was also to investigate if they fulfil the public health recommendations for physical activity.

Methods: 103 patients (93 women/10 men, mean age 51.5 (SD 15.9) years) with SLE according to the 1982 revised ACR criteria for SLE were involved. Physical activity was assessed with the short version of the International Physical Activity Questionnaire (IPAQ), which measure physical activity the last seven days. The IPAQ scientific group classify physical activity into the following categories: "inactive", "minimally active"(equal to public health recommendations) and "health enhancing physical activity". Those individuals who do not meet the criteria for the two latter categories are considered inactive.

Results: The patients reported that they were physically active in median 1666 (interquartile range 693; 3759) METs-minutes/week $(n=84)$. The patients answered that they were sitting in median 6 (interquartile range $4 ; 8$ ) hours/day the last week $(n=98) .59 .6 \%$ of the patients achieved a minimum of at least 600 METs-minutes/week, i.e. they were active 5 or more days with any combination of walking, moderate-intensity or vigorous intensity activities ("minimally active"). $17.9 \%$ achieved a minimum of at least 1500 METs-minutes/week, i.e. they were active on vigorous-intensity on at least 3 days or; achieved a minimum of at least 3000 METs-minutes/week, i.e. they were active 7 or more days on any combination of walking, moderate-intensity or vigorous intensity activities (" health enhancing physical activity"). $22.6 \%$ of the patients were "inactive".

Conclusions: In the investigated patients with SLE, the majority were "minimally active" according to IPAQ-categories, which is sufficiently physically active according to the minimum level of public health recommendations. However, only $1 / 5$ reached "health enhancing physical activity" category and $1 / 5$ were considered physically "inactive". Health professionals could use the short version of IPAQ to find out which patients with SLE need support in physical activity programmes.

\section{References:}

[1] Eriksson K et al. Physical activity in patients with systemic lupus erythematosus and matched controls. Scand J Rheumatol 2012; 41: 290-297.

[2] Ahn GE et al. Self-reported and objectively measured physical activity in adults with systemic lupus erythematosus. Arthritis Care \& Research 2015; 67:701-707.

Disclosure of Interest: None declared

DOI: 10.1136/annrheumdis-2017-eular.5359

\section{OP0257-HPR RELATIONSHIP BETWEEN SLEEP DISORDERS AND DISEASE ACTIVITY IN PATIENS WITH RHEUMATOID ARTHRITIS}

L. Villarreal $^{1}$, S. Henao ${ }^{2}$, D. Buitrago-Garcia ${ }^{3}$, P. Santos-Moreno ${ }^{4} .{ }^{1}$ Psychology and processes; ${ }^{2}$ Patient service; ${ }^{3}$ Epidemiology; ${ }^{4}$ Rheumatology, Biomab, Center for Rheumatoid Arthritis, Bogota, Bogota, Colombia

Background: Rheumatoid arthritis (RA) is the prevalent autoimmune inflammatory arthritis found in adults, with the worldwide prevalence ranging from $0.4 \%$ to $1.3 \%$ (1). Patients with this condition have permanent changes with different severity of arthritis deformities as well as functional disturbances; Studies had shown that every painful condition disturbs sleep, which can lead to mood and abilities disturbances (2).

Objectives: The aim of this study was to describe the socio-demographic profile and sleep disorders in RA patients from a specialized RA clinic in Colombia and relationship with disease activity.

Methods: A descriptive cross-sectional study was performed in a specialized clinic dedicated to care patients with rheumatoid arthritis (RA). Data was collected during our psychology consultation, through semi-structured interviews and non-probability sampling. Descriptive epidemiology was applied for continuous variables, using measures of central tendency and dispersion for categorical and qualitative variables by averages and percentages. We analyzed bivariate association with Pearson's $\mathrm{X}^{2}$.

Results: We included 1398 patients attending to our psychology consultation. Mean age was $55 \pm 8.80 \%$ were female and $20 \%$ male. Mean DAS28 was

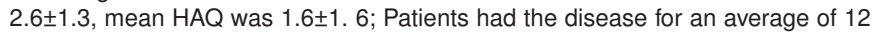
years $\pm 8 ; 41 \%$ of patients had comorbidities associated with non-autoimmune disease, $14 \%$ comorbidities related to autoimmune disease; $35 \%$ of our patients did not report other comorbidities. Most of patients were married $60 \%$, followed by divorced $19 \%$, single $14 \%$ and widowed $7 \%$. Regarding occupation $33 \%$ were employees, $25 \%$ were housekeepers or retired due to age, $12 \%$ were retired due to disabilities, and $3 \%$ unemployed. Of the total population $45 \%$ had elementary school, $32 \%$ high school, $8 \%$ college education, $7 \%$ graduate education and $7 \%$ were illiterate. $17 \%$ of patients lived alone. When the psychologist asked about sleep disorders $69 \%$ reported no to have any, $25 \%$ primary insomnia, $1 \%$ hypersomnia, $3 \%$ OSAS and $2 \%$ alterations on the circadian rhythm. Disease activity was statically associated with sleep disorders $(p<0.00)$.

Conclusions: Sleep problems are an important aspect to consider in a patient with RA and are correlated to disease activity; it is important to have a multidisciplinary care team for the patient with RA, including a psychologist that can manage this kind of illness in order to improve the life quality of patients.

References:

[1] Bautista-Molano W, Fernández-Avila D, Jiménez R, Cardozo R, Marín A, Soler $\mathrm{MdP}$, et al. Epidemiological Profile of Colombian Patients With Rheumatoid 